\title{
Ofloxacin MIC values and Mutation characteristics of Quinolones resistance determining region gene of Haemophilus influenzae isolates from the lower respiratory tract in Western Sichuan, China: Comparative study of children and adults
}

\section{Xiao Lei Wang ( $\square$ WXL3721@163.com )}

Affiliated Hospital of South West Jiao Tong University and the Third People's Hospital of Chengdu

\section{Jiang Xie}

Affiliated Hospital of South West Jiao Tong University and the Third People's Hospital of Chengdu

\section{Yuanbiao Guo}

Affiliated Hospital of South West Jiao Tong University and the Third People's Hospital of Chengdu

\section{Bingqing Zhu}

State Key Labratory for Infectious Disease Prevention and Control, National Institute for Communicable

Disease Control and Prevention, Chinese Center for Disease Control and Prevention

\section{Zhujun Shao}

State Key Labratory for Infectious Disease Prevention and Control, National Institue for

Communicatable Disease Control and Prevention, Chinese Center for Disease Control and Prevention

Huimin Guo

Affiliated Hospital of South West Jiao Tong University and the Third People's Hospital of Chengdu

Lili Yang

Affiliated Hospital of South West Jiao Tong University and the Third People's Hospital of Chengdu

Huawei Liu

Affiliated Hospital of South West Jiao Tong University and the Third People's Hospital of Chengdu

\section{Zhanhao Wang}

Affiliated Hospital of South West Jiao Tong university and the Third people's Hospital of Chengdu Jun $\mathrm{Hu}$

Affiliated Hospital of South West Jiao Tong University and the Third People's Hospital of Chengdu Lufei Huang

Affiliated Hospital of South West Jiao Tong University and the Third People's Hospital of Chengdu

\section{Research}


Keywords: Nontypeable Haemophilus influenzae, quinolone resistance determining region, gene mutation, amino acid substitution

Posted Date: May 6th, 2021

DOI: https://doi.org/10.21203/rs.3.rs-482903/v1

License: (c) (1) This work is licensed under a Creative Commons Attribution 4.0 International License. Read Full License 


\section{Abstract}

Background: In order to further investigate the trend of quinolone resistance and the gene variation characteristics of quinolone resistance determining region of non-typeable Haemophilus influenzae (NTHi) isolates from Chinese children's respiratory tract, We tracked the isolates of children in western Sichuan of China for ten years and compared them with adult groups in the same period.

Method: We monitored the MIC value of ofloxacin of NTHi isolates $(n=280)$ from lower respiratory tract secretions in children group $(n=57)$ during 2003 2004 and in whole age group $(n=223)$ during 2013 2014 in Western Sichuan, China. The amino acid sequences of QRDRs, gyrA, gyrB, parC and parE, were detected; and the relationship between amino acid substitutions $(A A S)$ of QRDRs and ofloxacin MIC value was analyzed. At the same time, the mutation trend of QRDRs gene in this region during the past ten years was compared analyzed.

Results: 1. All the strains $(\mathrm{n}=280)$ of $H$. influenzae included in the study were NTHi. No ofloxacin-resistant strains were found in 57 NTHi isolated from the children patient during 2003 2004. While strains with the minimum inhibitory concentration (MIC) value $\geq 0.5$ showed an upward trend in all age groups during 2013 2014. 2. The AAS are S84L, D88Y/N, A134V and E142K in gyrA, G399E, A400V, E469D and T472I in gyrB. S84R/I, S133A and N138S in parC and D364Y, A369T, R378C, A383T, G405S, D420N, A426V, V466M and S474N in parE. 3. Compared to the non-mutation group, the change of ofloxacin MIC values of the strains with S84L, D88Y/N and A134V in gyrA, A400V in gyrB and S84R/I , S133A in parC showed statistical significance ( $p \leq 0.05$ or approximate $p=0.05$ ); And the results of ordered multi-classification logistic analysis showed that the AAS gyrA-S84L (OR=139.824, 95\% $C /=55.730 ~ 350.811, p \rrbracket 0.001)$, gyrA$\mathrm{D} 88 \mathrm{Y} / \mathrm{N}(O R=28.950,95 \% C l=8.432 \sim 99.395, p \otimes 0.001)$, parC-S84R/I $(O R=102.789,95 \%$ $C l=31.851 \sim 331.713, p \llbracket 0.001)$, parC-S133A (OR=1.872, 95\% $C l=1.023 \sim 3.426, p=0.042)$ were risk factors for the increase of ofloxacin MIC value, respectively; the $A A S$ gyrB-A400V (OR $=0.517,95 \%$ $C l=0.322 \sim 0.831, p=0.006)$ was the factor affecting the decrease of ofloxacin MIC value. The gyrA's S84L, gyrA's D88Y/N, gyrB's A400V, parCs S84R/I and parCs S133A mutations were the main factors affecting the MIC value of ofloxacin of NTHi isolates in Western Sichuan, China, respectively. 4. The age group distribution of mutations S84L, D88Y/N and A134V in gyrA were significantly different $(p=0.013,0.034$ and 0.010 , respectively). The key mutation of multiple QRDRs of NTHi isolates in children in low age group is increasing rapidly. Along with increase of the mutation rate of S84L in gyrA and A400V in gyrB of isolates from $0 \sim 3$ yrs old group $\left(X^{2}=6.089, p=0.014 ; X^{2}=25.181, p<0.001\right)$, the ofloxacin MIC value increasing was statistically significant in the past ten years $(p=0.022)$.

\section{Background}

Fluoroquinolones are one of the most widely used antibiotics in the treatment of respiratory tract infections. Since the first discovery of quinolone-resistant strains in 1993[1], the quinolone resistant strains of $\mathrm{H}$. influenzae have gradually increased in many countries and regions. The mechanism of resistance to fluoroquinolones is considered to be related to the amino acid substitution of gyrA, gyrB, 
parC, and parE, the Quinolone Resistance-Determining Regions (QRDRs) of DNA cyclase type II topoisomerase and IV topoisomerase. Although quinolones have been banned in pediatrics after the listing of quinolones in China, our early research found that $H$. influenzae strains resistant to ciprofloxacin were still isolated from the respiratory tract of children and new borns [2]. To further explore the molecular mechanism of quinolones resistance and the variation characteristic of QRDRs genes of $H$. influenzae isolates from children's respiratory tract in China, we monitored the ofloxacin MIC values of the $H$. influenzae isolates from the lower respiratory tract secretions among all age groups during $2013 \sim 2014$ in Western Sichuan, China and sequenced the QRDRs of $g y r A$, gyrB, parC and parE. On this basis, the relationship between different $A A S$ patterns and ofloxacin MIC values was analyzed, and the gyrA, gyrB, parC and parE gene mutations of $H$. influenzae isolates in different age groups were compared. Partial of the epidemiological study of this work was presented at the 20th Annual Congress of Chinese Pediatric Society in Xiamen, China, 23 to 26 September 2015 and won the National Excellent Paper Award awarded by Chinese Pediatric Society.

\section{Methods}

\section{Strain source}

All strains were derived from two prospective epidemiological surveys in the Western Sichuan, China, $2003 \sim 2004$ and $2013 \sim 2014[3,4]$. The patient's age ranged from $0 \mathrm{~d}$ to $93 \mathrm{yrs}$, with a male to female ratio of $167: 113$. Group of $0 \sim 28 \mathrm{~d}: \mathrm{n}=19,6.79 \% ; 29 \mathrm{~d} \sim 1$ year group: $\mathrm{n}=110,39.29 \% ; 2 \sim 3$ yrs group: $\mathrm{n}=68,24.28 \% ; 4 \sim 6$ yrs group: $\mathrm{n}=31,11.07 \%$; adult group ( $>17 \mathrm{yrs}): \mathrm{n}=52,18.57 \% .57$ strains were isolated from 2003 to 2004, and 223 were isolated from 2013 to 2014. 228 strains in $0 \sim 17$ yrs group were respectively isolated from 180 cases $(78.95 \%)$ of bronchopneumonia and 19 cases $(8.33 \%)$ of neonatal pneumonia as well as 29 cases $(12.72 \%)$ of bronchiolitis; and the main diagnosis of patients in over 17 yrs group $(n=52)$ included 31 cases $(59.62 \%)$ of acute exacerbation of chronic obstructive pulmonary disease (AECOPD) and 18 cases (34.62\%) of pulmonary infection as well as 3 cases $(5.77 \%)$ of bronchitis.

The research program was approved by the Medical Ethics Review Committee of the institutional (the Approval No. CWSYLS-2013-R-1). Informed consent was obtained from patients or their parents or guardians according to the guidelines of the institutional review board on clinical samples.

\section{Strain Identification and DNA extraction}

Same as the literature [4].

\section{Antimicrobial susceptibility test}

The MIC value of ofloxacin was determined by broth dilution method, and the results were judged according to the breakpoint standard of Guidelines of CLSI 2016. The breakpoint of MIC to ofloxacin was $\leq 2 \mu \mathrm{g} / \mathrm{mL}$ as sensitive and $>2 \mu \mathrm{g} / \mathrm{mL}$ as resistant. Quality controlling was performed in each experiment by testing the MIC of reference $H$. influenzae strain ATCC49247 which was purchased from the Clinical Laboratory Center of the Ministry of Health of the People's Republic of China. 


\section{QRDRs gene sequencing}

The sequencing of the $g y r A$, gyrB, parC and parE gene were completed by Beijing Tianyi Huiyuan Life Science and Technology Co., Ltd. The primer sequence and base length of gyrA, gyrB, parC and parE genes are shown in Table 1[5]. PCR amplification reaction system: add the following ingredients to the $0.2 \mathrm{ml}$ centrifuge tube: genomic DNA $1.0 \mu \mathrm{l}, 10 \times$ Buffer (contain $2.5 \mathrm{mM} \mathrm{Mg}^{2+}$ ) $2.5 \mu \mathrm{l}$, Taq polymerase (5 $\mathrm{U} / \mu \mathrm{l}$, Polymerase from Dalian Bao Bio Takara Technology Co., Ltd.) $0.5 \mu \mathrm{l}$, dNTP (10 mM) $1.0 \mu \mathrm{l}$, Primer (+ -) $1 \mu \mathrm{l}(10 \mathrm{uM}), \mathrm{ddH}_{2} \mathrm{O} 0.5 \mu \mathrm{l}$, bulk volume $25 \mu \mathrm{l}$. Light and elastic mixing, instantaneous centrifugation collection of droplets from the tube wall to the tube bottom, PCR reaction on the PCR amplification instrument, the reaction parameters are as follows : $\left(94^{\circ} \mathrm{C} 5 \mathrm{~min}\right) * 1$ cycle; $\left(94^{\circ} \mathrm{C} 30 \mathrm{~s}, 58^{\circ} \mathrm{C} 30 \mathrm{~s}, 72^{\circ} \mathrm{C} 30\right.$ s) $\star 36$ cycles; $\left(72^{\circ} \mathrm{C} 7 \mathrm{~min}\right) * 1$ cycle. After the reaction, the $2 \mu \mathrm{l} \mathrm{PCR}$ product was detected by $1 \%$ agarose gel electrophoresis. The amplified PCR fragment was confirmed. PCR products were recovered by AxyPrepDNA gel recovery kit and the specific operation was carried out according to the kit specification. The purified PCR products were sequenced by sequenator (Applied Biosystems 3730-XL). Experimental instruments also included PCR instrument (Applied Biosystems 2720 Thermal cycler), Centrifuge (Eppendorf 5804R), GEL imaging system(UVP Biolmaging System). Nucleotide sequences are translated into amino acids using sequencer 4.1.0 sequencing analysis software. The 4.1.0 sequencing analysis software was used, and all obtained data are entered into Genbank, and the sequence was compared with that of $H$. influenzae Rd kw20; The accession numbers of gyrA gene, gyrB gene, parC gene and parE gene were HM113386.1, AJ508044.1, HG983321.1 and AJ508046.1, respectively.

Table 1

Primer sequences of PCR amplification of the gyrA, gyrB, parC and parE gene

\begin{tabular}{|c|c|c|c|}
\hline $\begin{array}{l}\text { Primers } \\
\text { of Gene }\end{array}$ & Sequence(5'-3') & $\begin{array}{l}\text { Product } \\
\text { length } \\
\text { (bp) }\end{array}$ & Procedure \\
\hline gyrA-F & CCGCCGCGTACTGTTCT & \multirow[t]{2}{*}{375} & \multirow{8}{*}{$\begin{array}{l}\left(94^{\circ} \mathrm{C} 5 \mathrm{~min}\right) * 1 \text { cycle; }\left(94^{\circ} \mathrm{C} 30 \mathrm{~s}, 58^{\circ} \mathrm{C} 30 \mathrm{~s},\right. \\
\left.72^{\circ} \mathrm{C} 30 \mathrm{~s}\right) * 36 \text { cycles; }\left(72^{\circ} \mathrm{C} 7 \mathrm{~min}\right) * 1 \mathrm{cycle} \\
4^{\circ} \mathrm{C} \infty\end{array}$} \\
\hline gyrA-R & CCATTTGCTAAAAGTGC & & \\
\hline gyrB-F & GGAAAATCCTGCAGATGC & \multirow[t]{2}{*}{445} & \\
\hline gyrB-R & AAGCAACGTACGGATGTG & & \\
\hline parC-F & TGGTTTAAAACCCGTTCA & \multirow[t]{2}{*}{370} & \\
\hline parC-R & AGCAGGTAAATATTGTGG & & \\
\hline parE-F & GAACGCTTATCATCACGCCA & \multirow[t]{2}{*}{471} & \\
\hline parE-R & AGCATCCGCGAGAATACAGA & & \\
\hline
\end{tabular}

\section{Data analysis}


The enumeration data were expressed as percentage (\%). There was a significant difference in $\chi 2$ or Fisher exact probability test between the two groups by using Statistical Software R (v3.3.1), and the difference was statistically significant with $p<0.05$. The MIC values of the two groups were compared by Rank Sum test. The median and quartile intervals were used to describe the center and the discrete trend, and $p<0.05$ is statistically significant for the difference. Two independent sample rank sum test (MannWhitney u test) and Multivariate Ordered Logistic Regression Analysis with spss20.0 Statistical Software for the data satisfying the condition. The independent variable is the mutation point of the gyrA gene, gyrB gene, parC gene and parE gene, respectively; and the dependent variable is the value of ofloxacin MIC value, in Multivariate Ordered Logistic Regression Analysis. The criteria is 0.05 , exclusion criteria is $0.10 ; p<0.05$ is statistically significant for the difference.

\section{Results}

\section{Strains serotype}

All the 280 strains of $H$. influenzae showed $p 6$ gene and fuck gene positive, while cap genes were negative. All $H$. influenzae strains were $N T H i$.

\section{Susceptibility of ofloxacin}

The results of antimicrobial susceptibility test by broth method showed that ofloxacin MICs of strains ( $\mathrm{n}$ = 57) during $2003 \sim 2004$ were $0.0156 \sim 1 \mu \mathrm{g} / \mathrm{mL}$, and no ofloxacin resistant strain was found; The frequency of ofloxacin resistant strains was 1.92\% (1/52) and the ofloxacin MIC value was $0.0156 \sim 16$ $\mu \mathrm{g} / \mathrm{mL}$ in adult group $(\mathrm{n}=52)$ during $2013 \sim 2014$; the ofloxacin MIC value was $0.0078 \sim 2 \mu \mathrm{g} / \mathrm{mL}$ in $0 \sim 3$ yrs group $(\mathrm{n}=142)$ and $0.0156 \sim 0.5 \mu \mathrm{g} / \mathrm{mL}$ in $4 \sim 6$ yrs group during $2013 \sim 2014$.

The results of Rank sum test showed that the ofloxacin MIC value increasing of $N T H i$ isolates $(n=142)$ in $0 \sim 3$ yrs group during $2013 \sim 2014$ was statistically significant compared with that of $2003 \sim 2004(\mathrm{n}=$ $55, p=0.022$ ). And the NTHi isolates with ofloxacin MIC $\geq 0.5 \mu \mathrm{g} / \mathrm{mL}$ also showed an increasing trend in all age groups during $2013 \sim 2014$, as shown in Table 2 and Table 3.

Table 2

The decade variation in MIC values of Ofloxacin of NTHi strains from 0-3yrs group (the results of Rank-Sum test)

\begin{tabular}{|lllllll|}
\hline Group & $\mathbf{n}$ & Median & \multicolumn{2}{l|}{ Percentiles } & Wilcoxon & $\boldsymbol{p}$ \\
\cline { 3 - 5 } & & & 25th & 75th & & \\
\hline 2003-2004 & 55 & 0.0313 & 0.0313 & 0.0313 & 11690.0 & 0.022 \\
\hline 2013-2014 & 142 & 0.0313 & 0.0156 & 0.0625 & & \\
\hline Notes: $p<0.05$ is statistically significant. & & & \\
\hline
\end{tabular}


Table 3

Proportion of strains with ofloxacin MIC $\geq 0.5$ strains in different Age groups (\%)

\begin{tabular}{|c|c|c|c|c|c|}
\hline Group & $\begin{array}{l}\text { MIC value } \\
\text { range }\end{array}$ & $M I C=0.5$ & $M I C=1$ & $M I C=2$ & $M I C=16$ \\
\hline \multicolumn{6}{|l|}{$2003 \sim 2004$} \\
\hline $\begin{array}{l}0 \sim 3 y r s \text { group }(n= \\
55)\end{array}$ & $0.0156 \sim 1$ & 0 & $1.81 \%(1 / 55)$ & 0 & 0 \\
\hline \multicolumn{6}{|l|}{$2013 \sim 2014$} \\
\hline $\begin{array}{l}0 \sim 3 y r s \text { group }(n= \\
142)\end{array}$ & $0.0078 \sim 2$ & $\begin{array}{l}4.93 \% \\
(7 / 142)\end{array}$ & $\begin{array}{l}2.82 \% \\
(4 / 142)\end{array}$ & $\begin{array}{l}1.41 \% \\
(2 / 142)\end{array}$ & 0 \\
\hline $\begin{array}{l}4 \sim 6 y r s \text { group }(n= \\
29)\end{array}$ & $0.0156 \sim 0.5$ & $8.31 \%(2 / 29)$ & 0 & 0 & 0 \\
\hline $\begin{array}{l}\geq 18 y \text { rs group }(n= \\
52)\end{array}$ & $0.0156 \sim 16$ & $9.62 \%(5 / 52)$ & $3.85 \%(2 / 52)$ & $1.92 \%(1 / 52)$ & $\begin{array}{l}1.92 \% \\
(1 / 52)\end{array}$ \\
\hline
\end{tabular}

\section{Mutation of QRDRs gene and its effect on ofloxacin MIC value}

The AAS in the gyrA gene were S84L, D88Y/N, A134V and E142K (gyrA-S84L, gyrA-D88Y/N, gyrA-A134V and gyrA-E142K). The AAS of gyrB gene were G399E, A400V, E469D and T472I (gyrB-G399E, gyrB-A400V, gyrB-E469D, gyrB-T472I). Mutations S84R/I and S133A and N138S appeared in the parC gene (parCS84R/I, parC-S133A and parC-N138S). The AAS in the parE gene were as follows: D364Y, A369T, R378C, A383T, G405S, D420N, A426V, V466M and S474N (parE-D364Y, parE-A369T, parE-R378C, parE-A383T, parE-G405S, parE-D420N, parE-A426V, parE-V466M and parE-S474N).

According to whether the above alleles were mutated, all strains were respectively divided into mutant group and non-mutant group, compared between the two groups using the Mann-Whitney $\mathrm{U}$ test. The effect of $A A S$ in QRDRs genes on the ofloxacin MIC value and the results of Mann-Whitney $U$ test were shown in Table 4. Compared to the non-mutation group, the change of ofloxacin MIC values of the strains with S84L, D88Y/N in gyrA, A400V in gyrB and S84R/I, S133A in parC showed statistical significance ( $p$ $\leq 0.05$ ); The changes of ofloxacin MIC values in the strains with gyrA-A134V, gyrA-E142K, parC-S133A, parC-N138S, parE-A369T, parE-A426V, respectively were at the critical value compared with non-mutation group $(p=0.053,0.054,0.07,0.072,0.064)$. There was no significant difference in the MIC value between the other $A A S$ groups and non-mutation group $(p>0.05)$. 
Table 4

The effect of amino acid substitution in QRDRs gene sequence on the MIC value of ofloxacin(MannWhitney U test, $\mathrm{n}=280$ )

\begin{tabular}{|c|c|c|c|}
\hline \multirow[t]{2}{*}{ Variation Site } & mutation strains & \multirow[t]{2}{*}{$\mathbf{U}$} & \multirow[t]{2}{*}{$p$} \\
\hline & $\mathrm{n}$ & & \\
\hline \multicolumn{4}{|l|}{ gyrA } \\
\hline S84L & 49 & 590.5 & $<0.001$ \\
\hline $\mathrm{D} 88 \mathrm{Y} / \mathrm{N}$ & 10 & 709 & 0.007 \\
\hline A134V & 5 & 314.5 & 0.029 \\
\hline E142K & 39 & 3823 & 0.053 \\
\hline \multicolumn{4}{|l|}{ gyrB } \\
\hline G399E & 1 & 137.5 & 0.986 \\
\hline A400V & 86 & 7179 & 0.05 \\
\hline E469D & 5 & 642 & 0.79 \\
\hline T472I & 1 & 73 & 0.529 \\
\hline \multicolumn{4}{|l|}{ parc } \\
\hline S84R/I & 19 & 404.5 & $<0.001$ \\
\hline S133A & 32 & 3126 & 0.054 \\
\hline N138S & 41 & 4006 & 0.07 \\
\hline \multicolumn{4}{|l|}{ parE } \\
\hline D364Y & 1 & 138 & 0.989 \\
\hline А369T & 2 & 83 & 0.072 \\
\hline R378C & 1 & 138 & 0.989 \\
\hline АЗ383Т & 1 & 138 & 0.989 \\
\hline G405S & 1 & 138 & 0.989 \\
\hline D420N & 3 & 273 & 0.979 \\
\hline A426V & 1 & 8 & 0.064 \\
\hline V466M & 11 & 1361 & 0.621 \\
\hline S474N & 7 & 741 & 0.28 \\
\hline
\end{tabular}


An ordered multi-classification logical regression analysis was performed on the 8 mutation sites with Mann-Whitney $\mathrm{U}$ test $p$ values less than 0.05 or close to 0.05 . The results of ordered multi-classification logistic analysis, with the $8 A A S$, respectively, as independent variable and ofloxacin MIC value as a dependent variable, showed that the AAS gyrA-S84L (OR=139.824, 95\% Cl=55.730 350.811, $p \llbracket 0.001)$, gyrA-D88Y $/ \mathrm{N}(O R=28.950,95 \% C l=8.432 \sim 99.395, p \otimes 0.001)$ and $p a r C-S 84 R / I(O R=102.789,95 \% C l=$ $31.851 \sim 331.713, p \llbracket 0.001)$ as well as parC-S133A $(O R=1.872,95 \% C l=1.023 \sim 3.426, p=0.042)$ were risk factors for the increase of ofloxacin MIC value, respectively; the AAS gyrB-A400V (OR=0.517, 95\% $\mathrm{Cl}$ $=0.322 \sim 0.831, p=0.006)$ was the factor affecting the decrease of ofloxacin MIC value. The above results are shown in Table 5.

Table 5

Effect of gene mutations of QRDRs on the variation of ofloxacin MIC value of NTHi isolates(by logistic regression analysis results)

\begin{tabular}{|c|c|c|c|c|c|c|c|}
\hline \multirow{2}{*}{$\begin{array}{l}\text { Variation } \\
\text { Site }\end{array}$} & \multirow[t]{2}{*}{ Estimate } & \multirow[t]{2}{*}{ OR } & \multirow{2}{*}{$\begin{array}{l}\text { Std. } \\
\text { Error }\end{array}$} & \multirow[t]{2}{*}{ Wald } & \multirow{2}{*}{$\begin{array}{l}P \\
\text { value }\end{array}$} & \multicolumn{2}{|c|}{ 95\% Confidence Interva } \\
\hline & & & & & & $\begin{array}{l}\text { Lower } \\
\text { Bound }\end{array}$ & $\begin{array}{l}\text { Upper } \\
\text { Bound }\end{array}$ \\
\hline gyrA-S84L & 4.940 & 139.824 & 0.469 & 110.809 & $<.001$ & 55.730 & 350.811 \\
\hline $\begin{array}{l}\text { gyrA- } \\
\text { D88Y/N }\end{array}$ & 3.366 & 28.950 & 0.629 & 28.597 & $\begin{array}{l}< \\
0.001\end{array}$ & 8.432 & 99.395 \\
\hline gyrA-A134V & 0.135 & 1.144 & 0.762 & 0.031 & 0.860 & 0.257 & 5.096 \\
\hline gyrA-E142K & -0.092 & 0.912 & 0.334 & 0.076 & 0.783 & 0.474 & 1.755 \\
\hline gyrB-A400V & -0.660 & 0.517 & 0.242 & 7.418 & 0.006 & 0.322 & 0.831 \\
\hline parC-S84R/I & 4.633 & 102.789 & 0.598 & 60.063 & $\begin{array}{l}< \\
0.001\end{array}$ & 31.851 & 331.713 \\
\hline parC-S133A & 0.627 & 1.872 & 0.308 & 4.133 & 0.042 & 1.023 & 3.426 \\
\hline parC-N138S & -0.449 & 0.638 & 0.351 & 1.642 & 0.200 & 0.321 & 1.269 \\
\hline
\end{tabular}

\section{Distribution trend of QRDRs gene mutation}

The results of $\chi 2$ test or Fisher exact probability analysis of the mutation rate of QRDRs genes of the isolates from $0 \sim 3$ yrs group in the past decade are shown in S1 table. The AAS patterns of the QRDRs genes isolated from $0 \sim 3 \mathrm{yrs}$ old $(n=142)$ were more diverse, with seven new mutation sites, including the gyrB-G399E and gyrB-E469D, and parE-D364Y, parE-A369T, parE-A383T, parE-G405S and parE-D420N, 
compared with $2003 \sim 2004(\mathrm{n}=55)$, as shown in S1 table; the results of Rank-Sum test show that the increase of gyrA-S84L, gyrB-A400V in $0 \sim 3$ yrs old group isolates from 2013 to 2014 was statistically $\operatorname{significant}\left(X^{2}=6.089,25.181 ; p=0.014, p<0.001\right)$. Meanwhile, the increase of ofloxacin MIC value in these isolates also shows statistical significance $(p=0.022)$, as shown in Table 2 . The trends of these eight QRDRs allele mutation, with the $p<0.05$ and $p$ close to 0.05 in $0 \sim 3$ yrs old group isolates throught by the Mann-Whitney $U$ test, are shown in Fig. 1.

The distribution of the strains with the 8 allelic mutations in the different age groups is shown in S2 table. The results of $\chi 2$ test or fisher exact probability method show that the distribution of D88Y/N and A134V mutation rate in $g y r A$ sequence as well as S84R/I mutation rate in parC sequence in different age group was significantly different $(p=0.027,0.029$ and 0.036$)$, respectively; The $p$ value of A400V variation in gyrB distribution difference in different age groups is also close to $0.05(p=0.052)$, but age group distribution of the other four loci had no statistical significance $(p>0.05)$. The distrubtion trende of QRDRs variation in neonatal group and $29 \mathrm{~d} \sim 3$ yrs group during the past decade is shown in Fig. 1 . Compared with the isolates from $2003 \sim 2004$, the gyrA-S84L, gyrA-E142K, gyrB-A400V, parC-S84R/I, parC-S133A variation of $29 \mathrm{~d} \sim 3$ yrs group isolates $(n=123)$ showed different increasing trend during $2013 \sim 2014$, among which gyrB-A400V variation increased the most. The gyrA-S84L, parC-S84R/I and parC-S133A variation of $0 \sim 28 \mathrm{~d}$ group isolates $(n=9)$ also increased by varying degrees.

The distribution trend of the above QRDRs mutations in different age group during $2013 \sim 2014$ is shown in Fig. 2. The gyrA-D88Y/N, gyrB-A400V, parC-S84R/I and parC-S133A variation rates of $0 \sim 28 \mathrm{~d}$ group isolates $(n=9)$ from $2013 \sim 2014$ were higher respectively than those in adults $(n=52)$. The gyrA-E142K, gyrB-A400V, parC-S84R/I and parC-S133A variation rates of isolates from $29 \mathrm{~d} \sim 1$ year group $(\mathrm{n}=64)$ in $2013 \sim 2014$ were higher at different degrees than those in adult groups.

The gyrA-E142K, gyrB-A400V, parC-S133A and parC-N138S variation rate of $2 \sim 3$ yrs isolates $(n=59)$ in $2013 \sim 2014$ were higher than those in adults at different degrees, among them, the rate of gyrB-A400V variation increased fastest.

The gyrA-S84L, gyrA-D88Y/N, gyrA-A134V, parC-S84R/I and parC-N138S variation rates of isolates from 4 $\sim 6$ yrs groups $(\mathrm{n}=31)$ in $2013 \sim 2014$ were lower than those in adult group, and the variation rate of gyrA-E142K, gyrA-E142K and parC-S133A was slightly higher than that in adult group.

The gyrB-A400V variation rates of isolates from groups $29 \mathrm{~d} \sim 1$ year $(\mathrm{n}=64)$ and $2 \sim 3 \mathrm{yrs}(\mathrm{n}=59)$ during $2013 \sim 2014$ were higher than those in adult groups $(n=52)$ in the same period, respectively. The gyrBA400V variation rate of $2 \sim 3 \mathrm{yrs}$ groups increased faster. The parC-S84R/I variation rates of $0 \sim 28 \mathrm{~d}$ group $(n=9)$ and $29 d \sim 1$ year group $(n=64)$ were also significantly higher than those of adult group $(n=$ 52). The parC-S133A variation rates of isolates from groups $0 \sim 28 \mathrm{~d}(\mathrm{n}=9), 29 \mathrm{~d} \sim 1$ year, $2 \sim 3 \mathrm{yrs}$ and 4 $\sim 6$ yrs were also higher than those in adult groups, but no statistical difference was shown.

\section{Discussion}


Through an all age group epidemiological survey in 2013 2014, we found that $H$. influenzae isolated from sputum from patients with lower respiratory tract infection in Western Sichuan Province were all $N T H i[3,4]$. Ofloxacin resistance rate was $1.98 \%(2 / 101)$ in the group of $\geq 18$ yrs old [6]. Although no resistant strain was found in the $0 \sim 17$ yrs old group [3], the result of the more accurate broth drug susceptibility test showed that the strains of ofloxacin MIC $\geq 0.5$ showed an increasing trend in all age groups. Indeed, $H$. influenzae isolated from all age groups is becoming less susceptibility to ofloxacin. Our findings are very similar to the results of a simultaneous study by shoji $S$ et al in Japan [7]. Unlike in China, tofloxacin (tosufloxacin) as one kind of fluoroquinolones has been approved for use in pediatric patients in Japan in 2010 [8]. As a result, it is not difficult to explain the possible reasons for the MIC value of ofloxacin isolated from respiratory secretions of Japanese children. Since 1993, when quinoloneresistant strains were first reported [9], the strains with reduced susceptibility to quinolones have been found in elderly patients in many countries and regions [10,11,17-20]. Because a group of fluoroquinolones, such as tosufloxacin, levofloxacin, moxifloxacin, garenoxacin and sitafloxacin, etc., have an excellent transfer rate to the lungs and show a strong antibacterial activity against most community-acquired pneumonia-causing pathogens, including S. pneumoniae, H. influenzae and Mycoplasma pneumoniae [9], they are called as 'respiratory quinolones' and are used as a first-line drug for adults with community-acquired pneumonia [12]. The widespread use of fluoroquinolones may induce the emergence of more drug-resistant strains in adult patients, but it does not explain why H. influenzae isolates with reduced ofloxacin sensitivity have been found frequently in Chinese children. The causes of high ofloxacin MIC value of NTHi strains in respiratory tract secretions of children in China should be highly concerned.

The mechanism of the quinolones killing bacteria involves the disruption of DNA replication of type II topoisomerase [13]. Type II topoisomerases are currently recognized to include DNA gyrase, which is responsible for the formation and elimination of supercoiled structures in DNA strands, and topoisomerase IV, which cuts and re-ligates tangled DNA during DNA replication [13]. Each of these enzymes is composed of two dimers of subunit types $A$ and $B$, which together form a tetramer. DNA gyrase is composed of gyrA and gyrB, topoisomerase IV comprises parC and parE [14]. The subunit $A$ (gyrA and parC) possesses DNA cutting and ligating activity, the subunit B (gyrB and parE) possesses ATPase activity [15]. Quinolones bind to the exposed double-stranded DNA and form the DNA-DNA gyrase-equinolone antibiotic complex, thereby preventing the re-ligation of DNA[16]. The substitutions of amino acids in each enzyme lead to the inhibition of the formation of antibiotic complex. In particular, mutations in the QRDRs are closely related to resistance [16]. Mutations in the QRDRs of $H$. influenzae have been demonstrated to occur in a stepwise manner with an increasing number of mutations yielding higher quinolone MIC value [17-21]. A sequence of nucleotides associated with quinolones resistance in the gyrA and parC gene sequences is known as the QRDRs. Among them, the QRDR region of the gyrA gene was composed of the $202 \sim 531$ st base at the fifth terminal of the gyrA gene (that is, the base encoding the $68 \sim 177$ th amino acid residues of the gyrA) and the $152 \sim 456$ th base at the terminal of the parC gene (that is, the amino acid residue at the $51 \sim 152 \mathrm{nd}$ position of the parC). Previously, quinoloneresistant $H$. influenzae with substitutions at ser-84 and asp-88 in $g y r A$, as well as those at gly-82, ser-84, 
glu-88 in parC have been reported [22, 23]. Kurt $\mathrm{F}$ et al [24] reported that in addition to the ser-84-leu and asp-88-asn in gyrA and the ser-84-ile in parC, the molecular characteristics of high levels of $H$. influenzae resistant to ciprofloxacin in southern Denmark also included K20R, asp-356-ala/thr-356-ala and met-481ile mutation of parC and glu-151-lys, ile-159-ala, D420N and ser-599-ala mutation of parE. Hisashi S et al. [25] showed that five amino acid substitutions in gyrA (at ser-84 and asp-88) and parC (at gly-82, ser-84 and glu-88) were closely related to the MIC by genetic transformation experiments. It was also observed that the degree of resistance is related to the number of the mutations [25].

We tried to analyze the effect of QRDRs amino acid substitution on the MIC value of ofloxacin by statistical method (orderly multiple classifications logistic regression analysis), and the results showed that the gyrA's S84L, gyrA's D88Y/N, gyrB's A400V, parCs S84R/I and parCs S133A mutations were the main factors affecting the MIC value of ofloxacin ( $p<0.001, p<0.001, p=0.006, p<0.001$ and $p<0.042)$, respectively; and the effect of the variation on MIC value of ofloxacin was 139.824 times, 28.950 times, 0.517 times, 102.789 times and 1.872 times of that of no mutation group, respectively. In addition, the results of Mann-Whitney U test showed that the gyrA's A134V and gyrA's E142K variations were significantly different from those of non-mutated group ofloxacin MIC $(p=0.029,0.053)$. However, the further ordered multiple classifications logistic regression analysis showed that the effect of the above two-locus variations on ofloxacin MIC value of the strain was not statistically significant $(p=0.860$, 0.783). We also found that the $A A S$ of parE sequence was complex, but the further statistical analysis indicated that the variation of parE sequence had no statistical significance on the MIC value of ofloxacin. Hisashi S et al.[25] verified previously the S84L, D88Y/N, E142K mutation of gyrA and the $\mathrm{S} 84 \mathrm{R} / \mathrm{I}$ and N138S mutation of parC by means of genetic transformation experiments, and the results were consistent with the results of this study. However, Hisashi $\mathrm{S}$ et al found that eight strains, containing gyrA-M121L and gyrA-E142K mutation, gyrB-A400V mutation, parC-S133A and parC-N138S mutations, the parE-V466M mutation, et al, showed a low range of moxifloxacin MIC $(0.015-0.06 \mu \mathrm{g} / \mathrm{ml})$ [25], which was not exactly consistent with our observation. According to the statistical results, we inferred that the QRDRs mutation sites affecting the MIC value of ofloxacin were gyrA's S84L (139.824 times), parCs S84R/I (102.789 times), gyrA's D88Y/N (28.950 times), parCs N138S (1.872 times) and gyrB's A400V ( 0.517 times), respectively. However, statistical analysis is not a substitute for further genetic transformation experiments and $\mathrm{Ka} / \mathrm{Ks}$ evolutionary analysis. The latter can help to clarify the real role of amino acid substitution in the mechanism of quinolone resistance emerging in the study and clarify the interrelationship between these amino acid substitutions.

In the past ten years, accompanied by a significant rising in the MIC value ofloxacin the gyrA's S84L and gyrB's A400V mutation rates of $N T H i$ isolates in the $0 \sim 3$ yrs old group in western Sichuan were significantly increased. It is suggested that not only a key mutation in the nucleic acid sequence in QRDRs of type II topoisomerase, but also the change of ATPase activity may be involved in the quinoloneresistance mechanism of $H$. influenzae strain. Through a detailed age grouping analysis, we found that the $g y r A$ 's D88Y/N, parCs S84R/I and parCs S133A mutation rates of NTHi isolates from group $0 \sim 28 \mathrm{~d}$ were much higher than those in adult group during 2013 2014; The gyrA's E142K, gyrB's A400V, parCs S84R/I and parCs S133A mutation rate in $29 \mathrm{~d} \sim 1$ year old group was higher than that of adult group at 
the same time; and the gyrA's E142K, gyrB's A400V and parCs S133A in $2 \sim 3$ yrs old group isolates continued to exceed adult levels; The gyrB's A400V and parCs S84R/I mutation rate of $4 \sim 6 \mathrm{yrs}$ old group strains was close to or below the adult group level, but the gyrA's E142K and parCs S133A mutation rates still exceeded the adult group level. While there were fewer cases in our two epidemiological surveys during $2003 \sim 2004$ and $2013 \sim 2014$ in the $0 \sim 28$ d groups and no large sample levels in the $29 \mathrm{~d} \sim$ 1 year old groups, the abnormal increase in the key mutation rate of QRDRs genes in these low age groups' isolates is very worrying in the context of the continuous ban of quinolones in children under 12 yrs of age in China. Besides the transmission factors of adult quinolone-resistant NTHi strains to children, the effect of quinolones residues in the environment and food chain on the resistance of NTHi isolates in children must also attract our attention. The genetic variation mechanism of quinolone resistance gene of NTHi strains in children is still worthy of our further exploration.

\section{Conclusions}

The gyrA's S84L, gyrA's D88Y/N, gyrB's A400V, parCs S84R/I and parCs S133A mutations were the main factors affecting the MIC value of ofloxacin of NTHi isolates in Western Sichuan, China, respectively. The key mutation of multiple QRDRs of NTHi isolates in children in low age group is increasing rapidly.

\section{Abbreviations}

NTHi, Nontypeable Haemophilus influenzae; QRDRs, the Quinolone Resistance-Determining Regions; MIC, minimum inhibitory concentration; yrs, years; LRTI, lower respiratory tract infections; CLSI, Institute of Clinical Laboratory Standards; PCR, Polymerase chain reaction; $A A S$, amino acid substitutions;

\section{Declarations}

\section{Acknowledgments}

Thanks to all the co-workers involved in the clinical cases of the Third People's Hospital of Chengdu (TPHC), the Women \& Children's Medical Center of Chengdu (WCMCD)and the People's Hospital of Deyang (PHD) as well as the Medical Center of Dujiangyan (MCD).

\section{Authors' Contributions}

Wang XL designed the experiment and wrote the first draft and together with Xie J, Guo YB and Shao ZJ further finalized the design. Zhu BQ, Hu J and Wang ZH planned the laboratory procedures. Guo HM and Huang LF conceptualized the statistical analyses. Yang LL and Liu HW performed sample collection. All authors had helped in revision and approved the final manuscript.

\section{Funding}


This study was funded by Science囚Technology Department of Sichuan Province (2013JY0121) and Science区Technology Department of Chengdu (2014-HM01-00272-SF). The funding bodies had no role in the design of the study and collection, analysis, and interpretation of data and in writing the manuscript.

\section{Availability of data and materials}

The datasets used and/or analysed during the current study are available from the corresponding author on reasonable request.

\section{Ethics approval and consent to participate}

The research program was approved by the Medical Ethics Review Committee of the institutional (the Approval No. CWSYLS-2013-R-1).

\section{Consent for publication}

Not applicable.

\section{Competing interests}

The authors declare that they have no competing interests.

\section{Author details}

${ }^{1}$ Affiliated Hospital of South West Jiao Tong University \& the Third People's Hospital of Chengdu, No.82 Qinglong Street, Qingyang District, Chengdu, Sichuan, China

${ }^{2}$ State Key Labratory for Infectious Disease Prevention and Control, National Institute for Communicatable Disease Control and Prevention, Chinese Center for Disease Control and Prevention, Beijing, China

\section{References}

1. Barriere SL, Hindler JA. Ciprofloxacin-resistant Haemophilus influenzae infection in a patient with chronic lung disease. Ann Pharmacother. 1993;27(3):309-310. doi:10.1177/106002809302700311

2. Zhang $L$, Wang $X L$, Huang $H$, et al. Analysis on variance of common pathogenic bacteria and drug resistance in children in Chengdu area from 2001 to 2006. Chin J Pediatr. 2007,45(12):927-931.

3. Hu J, Wang XL, Liu HW, et al. Multicenter prospective epidemiological studies on haemophilus influenzae infection in children hospitalized with lower respiratory tract infections. Chin J Pediatr. 2016.54(2)119-125. DOI:10.3760/cma.j.issn.0578-1310.2016.02.010

4. Wang XL, Xie J, Guo YB, et al. Lower respiratory tract isolates of non-typeable Haemophilus influenzae in Western Sichuan, China: Antimicrobial susceptibility, mechanisms of $\beta$-lactam 
resistance and decade changes. J Glob Antimicrob Resist. 2020;21:324-330.

doi:10.1016/j.jgar.2019.10.026

5. Satola SW, Schirmer PL, Farley MM. Complete sequence of the cap locus of Haemophilus influenzae serotype b and nonencapsulated b capsule-negative variants. Infect Immun. 2003; 71: 3639-44.

6. Liu HW,Wang XL,Guo YB,et al.Prospective epidemiological study on the adults of lower respiratory tract infection with haemophilus influenzae in Chengdu area. Chin J Lab.Med, 2017,40冈11囚:865-870. DOI:10.3760/cma.j.issn.1009-9158.2017.11.000

7. Shoji S, Takeaki W, Yuki Y, et al. Rise in Haemophilus influenzae With Reduced Quinolone Susceptibility and Development of a Simple Screening Method. Pediatr Infect Dis J. 2017;36:263266.

8. Ouchi K, Sunakawa K. Effect of new oral antimicrobial agents in outpatient treatment of pneumonia in children. Jpn J Antibiot. 2014;67:157-166.

9. Hooper DC. Mechanisms of fluoroquinolone resistance. Drug Resist Updat. 1999;2:38-55.

10. Pérez-Vázquez M, Román F, García-Cobos S, et al. Fluoroquinolone resistance in Haemophilus influenzaeis associated with hypermutability. Antimicrob Agents Chemother. 2007;51:1566-1569.

11. Kuo SC, Chen PC, Shiau YR, et al. Levofloxacin-resistant Haemophilus influenzae, Taiwan, 20042010. Emerg Infect Dis. 2014;20:1386-1390.

12. The Committee for the Japanese Respiratory Society Guidelines in Management of Respiratory Infections. The Japanese Respiratory Society guideline for the management of community-acquired pneumonia in adults. Respirology. 2006;11(Suppl. 3):79-133.

13. Hooper DC, Jacoby GA. Mechanisms of drug resistance: quinolone resistance. Ann N Y Acad Sci .2015;1354:12-31.

14. Gubaev A, Klostermeier D. Potassium ions are required for nucleotide-induced closure of gyrase Ngate. J Biol Chem. 2012;287:10916-21.

15. Gubaev A, Klostermeier D. DNA-induced narrowing of the gyrase N-gate coordinates T-segment capture and strand passage. Proc Natl Acad Sci USA. 2011;108:14085-90.

16. Drlica K, Hiasa H, Kerns R, Malik M, Mustaev A, Zhao X. Quinolones: action and resistance updated. Curr Top Med Chem. 2009;9:981-98.

17. Puig C, Tirado-Vélez JM, Calatayud L, Tubau F, Garmendia J, Ardanuy C, et al. Molecular characterization of fluoroquinolone resistance in nontypeable Haemophilus influenzae clinical isolates. Antimicrob Agents Chemother. 2015;59:461-6.

18. Pe'rez-Va'zquez M, Roma'n F, Aracil B, Canto'n R, Campos J. Laboratory detection of Haemophilus influenzae with decreased susceptibility to nalidixic acid, ciprofloxacin, levofloxacin, and moxifloxacin due to GyrA and ParC mutations. J Clin Microbiol. 2004;42:1185-91.

19. Li X, Mariano N, Rahal JJ, Urban CM, Drlica K. Quinolone-resistant Haemophilus influenzae in a longterm-care facility: nucleotide sequence characterization of alterations in the genes encoding DNA gyrase and DNA topoisomerase IV. Antimicrob Agents Chemother. 2004;48:3570-2. 
20. Georgiou M, Mun oz R, Roma'n F, Canto'n R, Go'mez-Lus R,Campos J, et al. Ciprofloxacin-resistant Haemophilus influenzae strains possess mutations in analogous positions of GyrA and ParC. Antimicrob Agents Chemother. 1996;40:1741-4.

21. Shoji H, Shirakura T, Fukuchi K, Takuma T, Hanaki H, Tanaka K, et al. A molecular analysis of quinolone-resistant Haemophilus influenzae: validation of the mutations in quinolone resistancedetermining regions. J Infect Chemother. 2014;20:250-5.

22. Hirakata Y, Ohmori K, Mikuriya M, Saika T, Matsuzaki K, Hasegawa M. Antimicrobial activities of piperacillin-tazobactam against Haemophilus influenzae isolates, including beta-lactamasenegativeampicillin-resistant and betalactamase-positive amoxicillin-clavulanate-resistant isolates, and mutations in their quinolone resistance-determining regions. Antimicrob Agents Chemother. 2009;53:4225-30.

23. Yokota S, Ohkoshi Y, Sato K, Fujii N. Emergence of fluoroquinolone-resistant Haemophilus influenzae strains among elderly patients but not among children. J Clin Microbiol. 2008;46:361-5.

24. Kurt F, Gitte N H, Marc S,et al.Molecular characterisation of the clonal emergence of high-level ciprofloxacin-mono resistant Haemophilus influenzae in the Region of Southern Denmark. J Global Antimicro Resistance. 2016, 5: 67-70.

25. Hisashi S, Tetsuro S, Kunihiko F,et al.Amolecular analysis of quinolone-resistant Haemophilus influenzae:Validation of the mutations in Quinolone Resistance-Determining Regions. J Infect Chemother 2014,20 : 250-255.

\section{Tables}

Table 1 Primer sequences of PCR amplification of the gyrA, gyrB, parC and parE gene

\begin{tabular}{|c|c|c|c|}
\hline $\begin{array}{l}\text { Primers } \\
\text { of Gene }\end{array}$ & Sequence $\left(5^{\prime}-3^{\prime}\right)$ & $\begin{array}{l}\text { Product } \\
\text { length } \\
\text { (bp) }\end{array}$ & Procedure \\
\hline gyrA-F & CCGCCGCGTACTGTTCT & 375 & \multirow{8}{*}{$\begin{array}{l}\left(94^{\circ} \mathrm{C} 5 \mathrm{~min}\right) * 1 \mathrm{cycle} ;\left(94^{\circ} \mathrm{C} 30 \mathrm{~s}, 58^{\circ} \mathrm{C}\right. \\
\left.30 \mathrm{~s}, 72^{\circ} \mathrm{C} 30 \mathrm{~s}\right) * 36 \text { cycles; }\left(72^{\circ} \mathrm{C} 7 \mathrm{~min}\right) * 1 \\
\text { cycle; } 4^{\circ} \mathrm{C} \infty .\end{array}$} \\
\hline gyrA-R & CCATTTGCTAAAAGTGC & & \\
\hline gyrB-F & GGAAAATCCTGCAGATGC & 445 & \\
\hline gyrB-R & AAGCAACGTACGGATGTG & & \\
\hline parC-F & TGGTTTAAAACCCGTTCA & 370 & \\
\hline parC-R & AGCAGGTAAATATTGTGG & & \\
\hline parE-F & GAACGCTTATCATCACGCCA & 471 & \\
\hline parE-R & AGCATCCGCGAGAATACAGA & & \\
\hline
\end{tabular}


Table 2 The decade variation in MIC values of Ofloxacin of NTHi strains from 0-3yrs group (the results of Rank-Sum test)

\begin{tabular}{|c|c|c|c|c|c|c|}
\hline \multirow[t]{2}{*}{ Group } & \multirow[t]{2}{*}{$\mathrm{n}$} & \multirow[t]{2}{*}{ Median } & \multicolumn{2}{|c|}{ Percentiles } & \multirow[t]{2}{*}{ Wilcoxon } & \multirow[t]{2}{*}{$p$} \\
\hline & & & 25 th & 75th & & \\
\hline 2003-2004 & 55 & 0.0313 & 0.0313 & 0.0313 & 11690.0 & $\overline{0.022}$ \\
\hline 2013-2014 & 142 & 0.0313 & 0.0156 & 0.0625 & & \\
\hline
\end{tabular}

Notes: $p<0.05$ is statistically significant.

Table 3 Proportion of strains with ofloxacin MIC $\geq 0.5$ strains in different Age groups (\%)

\begin{tabular}{|c|c|c|c|c|c|}
\hline Group & $\begin{array}{l}\text { MIC value } \\
\text { range }\end{array}$ & $\mathrm{MIC}=0.5$ & $\mathrm{MIC}=1$ & $\mathrm{MIC}=2$ & $\mathrm{MIC}=16$ \\
\hline $2003 \sim 2004$ & & & & & \\
\hline $\begin{array}{c}0 \sim 3 \text { yrs group } \\
(\mathrm{n}=55)\end{array}$ & $0.0156 \sim 1$ & 0 & $\begin{array}{l}1.81 \% \\
(1 / 55)\end{array}$ & 0 & 0 \\
\hline $2013 \sim 2014$ & & & & & \\
\hline $\begin{array}{c}0 \sim 3 \mathrm{yrs} \\
\text { group }(\mathrm{n}=142)\end{array}$ & $0.0078 \sim 2$ & $\begin{array}{l}4.93 \% \\
(7 / 142)\end{array}$ & $\begin{array}{c}2.82 \% \\
(4 / 142)\end{array}$ & $\begin{array}{l}1.41 \% \\
(2 / 142)\end{array}$ & 0 \\
\hline $\begin{array}{c}4 \sim 6 y r s \\
\text { group }(n=29)\end{array}$ & $0.0156 \sim 0.5$ & $\begin{array}{l}8.31 \% \\
(2 / 29)\end{array}$ & 0 & 0 & 0 \\
\hline $\begin{array}{c}\geq 18 \mathrm{yrs} \\
\text { group }(n=52)\end{array}$ & $0.0156 \sim 16$ & $\begin{array}{l}9.62 \% \\
(5 / 52)\end{array}$ & $\begin{array}{l}3.85 \% \\
(2 / 52)\end{array}$ & $\begin{array}{l}1.92 \% \\
(1 / 52)\end{array}$ & $\begin{array}{l}1.92 \% \\
(1 / 52)\end{array}$ \\
\hline
\end{tabular}

Note: 55 of 57 cases during 2003 to 2004 belong to $0 \sim 3$ years group ,and 2 cases are of $4 \sim 6$ years old. 
Table 4 The effect of amino acid substitution in QRDRs gene sequence on the MIC value of ofloxacin (Mann-Whitney U test, $\mathrm{n}=\mathbf{2 8 0}$ )

\begin{tabular}{|c|c|c|c|}
\hline & \multirow{2}{*}{\multicolumn{3}{|c|}{$\mathrm{n}$}} \\
\hline & & & \\
\hline \multicolumn{4}{|l|}{ gyrA } \\
\hline S84L & 49 & 590.5 & $<0.001$ \\
\hline D88Y/N & 10 & 709 & 0.007 \\
\hline A134V & 5 & 314.5 & 0.029 \\
\hline E142K & 39 & 3823 & 0.053 \\
\hline \multicolumn{4}{|l|}{ gyrB } \\
\hline G399E & 1 & 137.5 & 0.986 \\
\hline $\mathrm{A} 400 \mathrm{~V}$ & 86 & 7179 & 0.05 \\
\hline E469D & 5 & 642 & 0.79 \\
\hline T472I & 1 & 73 & 0.529 \\
\hline \multicolumn{4}{|l|}{ parC } \\
\hline $\mathrm{S} 84 \mathrm{R} / \mathrm{I}$ & 19 & 404.5 & $<0.001$ \\
\hline S133A & 32 & 3126 & 0.054 \\
\hline N138S & 41 & 4006 & 0.07 \\
\hline \multicolumn{4}{|l|}{ parE } \\
\hline D364Y & 1 & 138 & 0.989 \\
\hline A369T & 2 & 83 & 0.072 \\
\hline R378C & 1 & 138 & 0.989 \\
\hline A383T & 1 & 138 & 0.989 \\
\hline G405S & 1 & 138 & 0.989 \\
\hline D420N & 3 & 273 & 0.979 \\
\hline $\mathrm{A} 426 \mathrm{~V}$ & 1 & 8 & 0.064 \\
\hline V466M & 11 & 1361 & 0.621 \\
\hline S474N & 7 & 741 & 0.28 \\
\hline
\end{tabular}

notice: Amino acid abbreviation A-Ala, G-Gly, V-Val, L-Leu, I-Ile, M-Met, S-Ser, T-Thr, N-Asn, Q-Gln, D-Asp, E-Glu, K-Lys, R-Lys, C-Cys, Y-Tyr. $p<0.05$ is statistically significant.

Table 5 Effect of gene mutations of QRDRs on the variation of ofloxacin MIC value of NTHi isolates (by logistic regression analysis results)

Variation Site Estimate OR Std. Error Wald Pvalue 95\% Confidence Interval Lower Bound Upper Bound

\begin{tabular}{lrrrrrrr}
\hline gyrA-S84L & 4.940 & 139.824 & 0.469 & 110.809 & $<0.001$ & 55.730 & 350.811 \\
\hline gyrA-D88Y/N & 3.366 & 28.950 & 0.629 & 28.597 & $<0.001$ & 8.432 & 99.395 \\
\hline gyrA-A134V & 0.135 & 1.144 & 0.762 & 0.031 & 0.860 & 0.257 & 5.096 \\
\hline gyrA-E142K & -0.092 & 0.912 & 0.334 & 0.076 & 0.783 & 0.474 & 1.755 \\
\hline & & & & & & & \\
gyrB-A400V & -0.660 & 0.517 & 0.242 & 7.418 & 0.006 & 0.322 & 0.831 \\
\hline & & & & & & & \\
\hline parC-S84R/I & 4.633 & 102.789 & 0.598 & $60.063<0.001$ & 31.851 & 331.713 \\
parC-S133A & 0.627 & 1.872 & 0.308 & 4.133 & 0.042 & 1.023 & 3.426 \\
\hline parC-N138S & -0.449 & 0.638 & 0.351 & 1.642 & 0.200 & 0.321 & 1.269 \\
\hline
\end{tabular}




\section{Figures}

Fig 1 The distrubtion trende of QRDRs variation in neonatal group and $29 \mathrm{~d} \sim 3 \mathrm{yrs}$ group during the past decade

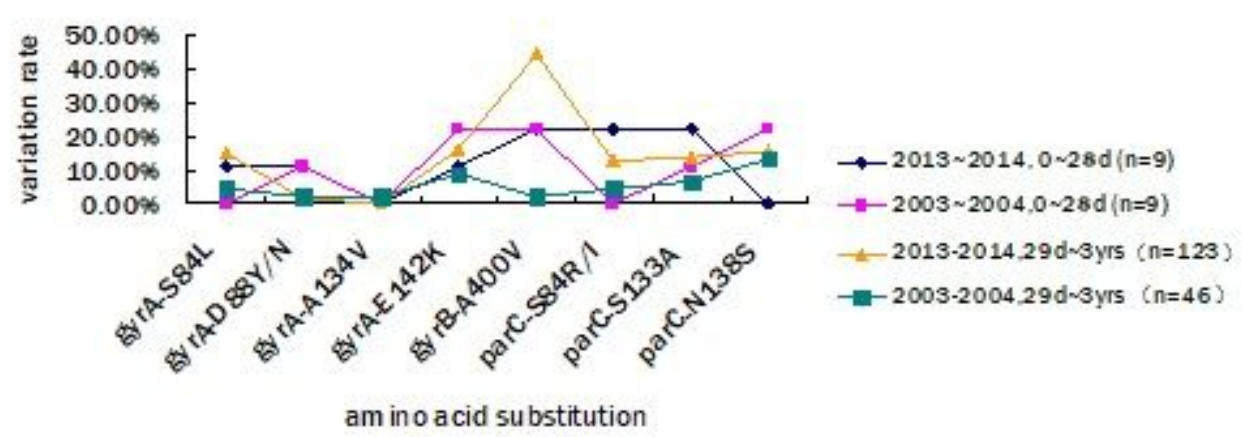

\section{Figure 1}

(caption in figure)

Fig 2 comparison of QFDFs gene variation in different age group during 2013 2014

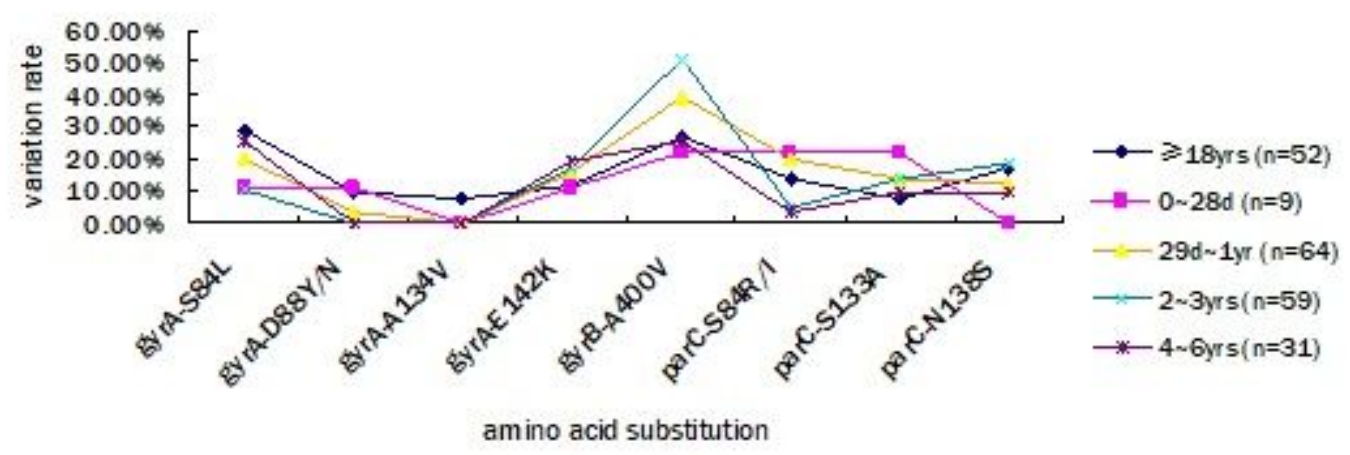

Figure 2

(caption in figure)

\section{Supplementary Files}

This is a list of supplementary files associated with this preprint. Click to download.

- S1Table.doc

- S2Table.doc 